\title{
Numerical Simulation of Incompressible Viscous Flow Using an Implicit Fractional Step Method on Collocated Grid
}

\author{
Li-wei Song ${ }^{1}$ \\ ${ }^{1}$ School of Aeronautical Science and Engineering \\ Beihang University \\ Beijing, China
}

\author{
Song-ping $\mathrm{Wu}^{1,2,3}$ \\ ${ }^{2}$ Key Laboratory of Fluid Mechanics of Ministry of Education \\ Beihang University \\ Beijing, China \\ ${ }^{3}$ National Laboratory for Computational Fluid Dynamics \\ Beijing, China
}

\begin{abstract}
In this paper, incompressible viscous flow is numerically analyzed by solving unsteady two-dimensional Navier-Stokes equations. Simulations are carried out by using an implicit fractional step method which consists three sub-steps: calculation of intermediate velocity, solution of the pressure correction equation, and updating of the pressure and velocity. A collocated grid system is employed rather than a staggered grid system because of the simplicity and ease of extension to threedimensional analysis. Several benchmark flows, including the liddriven cavity flow, the flow past a square cylinder and the flow around a circular cylinder were computed and validated against previous simulations or experiments to prove the accuracy and effectiveness of the present method. In this latter case, an immersed boundary method is used to handle the embedded cylinder boundary.
\end{abstract}

Keywords-Numerical sumulation; Computational Fluid Dynamics; incompressible viscous flow; fractional step method; collocated grid

\section{INTRODUCTION}

Numerical simulation of fluid flow has been a major topic of research not only for scientist but also for engineer in the past few decades. Computational fluid dynamics (CFD) involves describing the fluid flow in terms of mathematical models that consist of governing equations in the form of partial differential equations. Numerical methods for incompressible viscous flow are a major part of the rapidly growing field CFD. A major difficulty for the numerical simulation of incompressible flow is that the velocity and the pressure are coupled by the incompressibility constraint. Fractional step method for the incompressible Navier-Stokes equations gained its popularity due to its computational efficiency, achieved by decoupling of the velocity and the pressure.

Staggered and collocated approached are both acceptable and common approaches for solving fluid flow problems. In

Project supported by the National Natural Science Foundation of China (No. 91016006). terms of coding and application, each of them has some relative merits and advantages. However the most apparent difference between these two approaches is the capability of collocated grid arrangement in dandling the complex geometries.

In the present paper, an implicit fractional step method on collocated grid is used to solve incompressible viscous flow. The detailed description of the present method will be given below followed by some verifying and validating examples.

\section{NUMERICAL MATHOD}

In the present work the laminar two dimensional flow field of incompressible fluid is governed by the Navier-Stokes equations. The dimensionless unsteady incompressible NavierStokes equations in primitive variables can be written as

$$
\begin{aligned}
& \frac{\partial \mathbf{u}}{\partial t}+\mathbf{u} \cdot \nabla \mathbf{u}=-\nabla p+\frac{1}{\operatorname{Re}} \nabla^{2} \mathbf{u} \\
& \nabla \cdot \mathbf{u}=0
\end{aligned}
$$

where $\mathbf{u}$ and $p$ are the non-dimensional velocity vector and kinetic pressure, respectively, and $\mathrm{Re}$ is the Reynolds number.

The Navier-Stokes equations are discretized using a cellcentered, collocated arrangement of the primitive variables $\left(u_{i}, p\right)$. In addition to the cell-center velocities $\left(u_{i}\right)$, the facecenter velocities, $U_{i}$, are computed. The equations are integrated in time suing the fractional step method of Van-Kan $[1,2]$ which consists three sub-steps.

In the first sub-step of the method, a modified momentum equation is solved and an intermediate velocity $\mathbf{u}^{*}$ obtained. A second-order, Adams-Bashforth scheme is employed for the convective terms while the diffusion terms are discretized using an implicit Crank-Nicolson scheme which eliminates the viscous stability constraint. In this sub-step, the following modified momentum equation is solved at the cell-nodes

$$
\frac{u_{i}^{*}-u_{i}^{n}}{\Delta t}+\frac{1}{2}\left[3 N_{i}^{n}-N_{i}^{n-1}\right]=-\frac{\delta p^{n}}{\delta x_{i}}+\frac{1}{2}\left(D_{i}^{*}+D_{i}^{n}\right)
$$




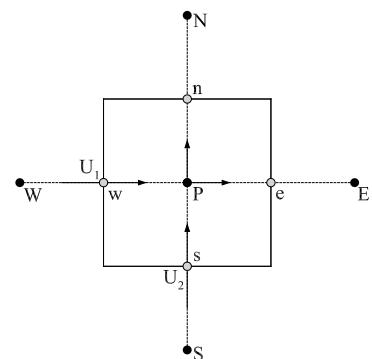

Figure 1. Schematie describing the naming convention and location of velocity components employed in the spatial discretization of the governing equations.

This equation is solved using a line-SOR scheme. Subsequently, face-center velocities at this intermediate step $U^{*}$ are computed by averaging the corresponding values at the grid nodes

$$
\begin{aligned}
& \tilde{u}_{i}=u_{i}^{*}+\Delta t\left(\frac{\delta p^{n}}{\delta x_{i}}\right)_{c c} \\
& \tilde{U}_{1}=\gamma_{w} \tilde{u}_{1 P}+\left(1-\gamma_{w}\right) \tilde{u}_{1 W} \\
& \tilde{U}_{2}=\gamma_{s} \tilde{u}_{1 P}+\left(1-\gamma_{s}\right) \tilde{u}_{1 S} \\
& U_{i}^{*}=\tilde{U}_{i}-\Delta t\left(\frac{\delta p^{n}}{\delta x_{i}}\right)_{f c}
\end{aligned}
$$

where $\gamma_{w}$ and $\gamma_{s}$ are the weights corresponding to linear interpolation for the west and south face velocity components respectively. Furthermore, $c c$ and $f c$ denote gradients computed at cell-centers and face-centers, respectively. This procedure is necessary to eliminate odd-even decoupling that usually occurs with non-staggered methods and which leads to large pressure variations in space.

The second sub-step requires the solution of the pressure correction equation

$$
\frac{u_{i}^{n+1}-u_{i}^{*}}{\Delta t}=-\frac{\delta p^{\prime}}{\delta x_{i}}
$$

which is solved with the constraint that the final velocity $u_{i}^{n+1}$ be divergence-free. This gives the following Poisson equation for the pressure correction

$$
\frac{\delta}{\delta x_{i}}\left(\frac{\delta p^{\prime}}{\delta x_{i}}\right)=\frac{1}{\Delta t} \frac{\delta U_{i}^{*}}{\delta X_{i}}
$$

and a Neumann boundary condition imposed on this pressure correction at all boundaries.

Once the pressure correction is obtained, the pressure and velocity are updated as

$$
\begin{aligned}
& p^{n+1}=p^{n}+p^{\prime} \\
& u_{i}^{n+1}=u_{i}^{*}-\Delta t\left(\frac{\delta p^{\prime}}{\delta x_{i}}\right)_{c c}
\end{aligned}
$$

$$
U_{i}^{n+1}=U_{i}^{*}-\Delta t\left(\frac{\delta p^{\prime}}{\delta x_{i}}\right)_{f c}
$$

The above collocated scheme is simpler to implement than a conventional staggered mesh scheme and when coupled with central-difference spatial scheme, it leads to a numerical discretization that has good discrete kinetic energy conservation properties making it suitable and robust for simulating relatively high (up to at least $\mathrm{O}\left(10^{4}\right)$ ) Reynolds number flows without the need for artificial dissipation or upwinding.

\section{RESULTS AND DISCUSSION}

In this section we give extensive numerical evidence of the versatility and accuracy of the method by computing a number of steady and unsteady, incompressible viscous flow problems. All the simulations are performed with CFL number within the range 0.5-0.9.

\section{A. Lid-driven cavity flow}

The two-dimensional lid-driven cavity flow is a simple incompressible viscous flow that has many features suitable for testing the performance of a numerical method, including the primary and the secondary vortices, wall boundary layers, and flow separation and reattachment. The flow was studied numerically by Ghia et al. [3], who reported accurate solutions to the steady flow solution over a range of Reynolds numbers.

Lid-driven cavity flows with uniform lid velocity at the Reynolds numbers of 100, 1000, and 3200 (based on the lid velocity) were simulated to verify the present flow solver and have been the subject of a numerical study by Meng-Hsuan Chung [4]. The geometry, initial conditions, and boundary conditions are shown in Fig. 2. A $128 \times 128$ uniform grid was used in the computations. Fig. 3 presents the steady-state streamlines at the three Reynolds numbers.

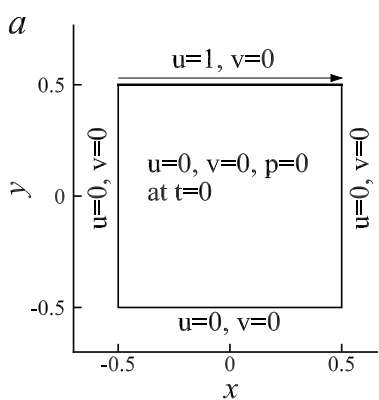

$b$

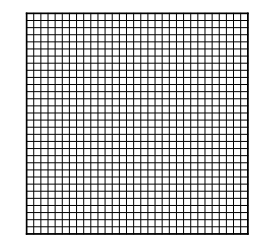

Figure 2. (a) Geometry, initial conditions, boundary conditions and (b) uniform grid of the lid-driven cavity flow.

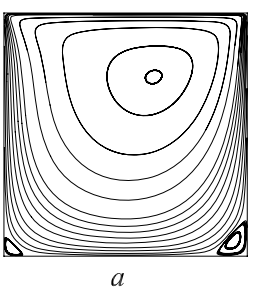

Figure 3. Steady-state streamlines of the lid-driven cavity flow with uniform lid velocity. (a) $R e=100$, (b) $R e=1000$ and (b) $R e=3200$. 


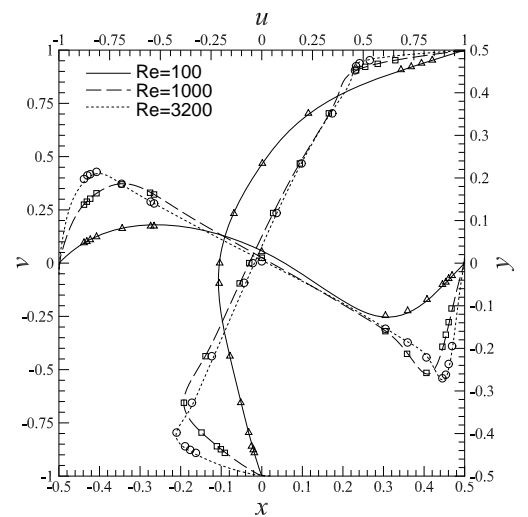

Figure 4. Steady-state center-line velocity profiles $u(0, y)$ and $v(x, 0)$ of the liddriven cavity flow with uniform lid velocity for $\mathrm{Re}=100, \mathrm{Re}=1000$ and $\mathrm{Re}=3200$. Comparison between the present computation (line) and Ghia et al. [3] (symbol).

Fig. 4 shows the steady-state $u$ and $v$ profiles along the vertical and horizontal center lines, respectively. The result of Ghia et al. [3] is plotted for comparison. It can be seen that the agreement is good. Table I lists the locations of the primary and the first vortices predicted by the present implicit fractional step method on collocated grid and makes a comparison with Ghia et al. [3]. Therefore, the code for the implicit fractional step method is preliminarily verified.

TABLE I. LOCATION OF PRIMARY AND FIRST VORTICES OF LID-DRIVEN CAVITY FLOW BY THE PRESENT METHOD AND IN THE WORK OF GHIA ET AL. [3], RESPETIVELY, BL: BOTTOM LEFT CORNER, BR: BOTTOM RIGHT CORNER, TL: LEFT CORNER.

\begin{tabular}{|c|c|c|c|c|c|}
\hline Source & Re & Primary & BL & BR & TL \\
\hline Ghia[? & \multirow{2}{*}{100} & 4 & $-0.4678,-0.4609$ & $0.4453,-0.4375$ & - \\
\hline Present & & $0.1157,0.2372$ & $-0.4652,-0.4651$ & $0.4426,-0.4379$ & - \\
\hline Ghia[3] & \multirow{2}{*}{1000} & $0.0313,0.0625$ & $-0.4141,-0.4219$ & $0.3594,-0.3096$ & - \\
\hline Present & & $0.0309,0.0657$ & $-0.4166,-0.4221$ & $0.3632,-0.3875$ & - \\
\hline Ghia[3] & \multirow{2}{*}{3200} & $0.0165,0.0469$ & $-0.4141,-0.3906$ & $0.3125,-0.4141$ & $-0.4453,0.3984$ \\
\hline Present & & $0.0177,0.0410$ & $-0.4190,-0.3809$ & $0.3196,-0.4155$ & $-0.4449,0.3988$ \\
\hline
\end{tabular}

\section{B. Flow around a square cylinder}

Flow around a square cylinder is of practical importance in many fields of engineering and science. There have been numerous detailed studies of flow past a square cylinder. Flow around a square cylinder is a typical model to validate the performance of numerical methods for solution of incompressible viscous Navier-Stokes equations. The flow structure has been investigated experimentally and numerically.

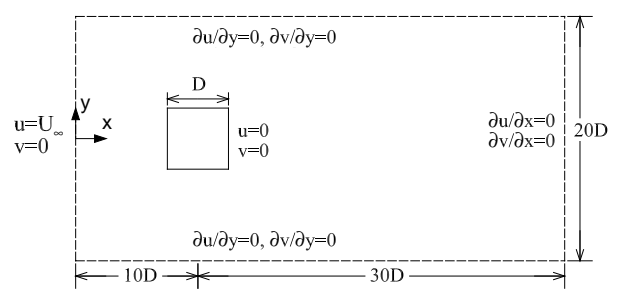

Figure 5. Computational domain and boundary conditions for flow around a square cylinder.
In this case study, a rectangular domain was used to simulate the flow past a stationary square cylinder. The computational domain used by Shen and Chan [5] is shown in Fig. 5, with the coordinate system and boundary conditions displayed in the figure. A $40 D \times 20 D$ domain is discretized into $295 \times 240$ non-uniform grids with the minimum grid size of $\Delta x=\Delta y=0.025 D$ being employed near the cylinder. The following flow variables, including the drag coefficient $C_{D}=-$ $F_{x} / 0.5 \rho U_{\infty} D$, lift coefficient $C_{D}=-F_{y} / 0.5 \rho U_{\infty} D$ and Strouhal number $S t=f D / U_{\infty}$, where $f$ is the vortex-shedding frequency determined from the time series $C_{L}(t)$. $C_{L, r m s}$ is the root-meansquare lift coefficient.
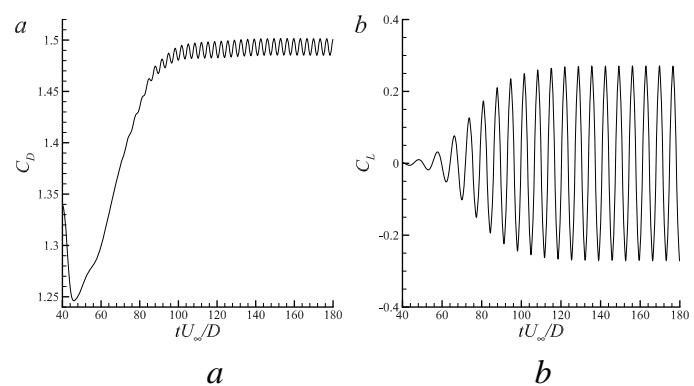

Figure 6. Time-dependent acting force coefficients: (a) drag force and (b) lift force.

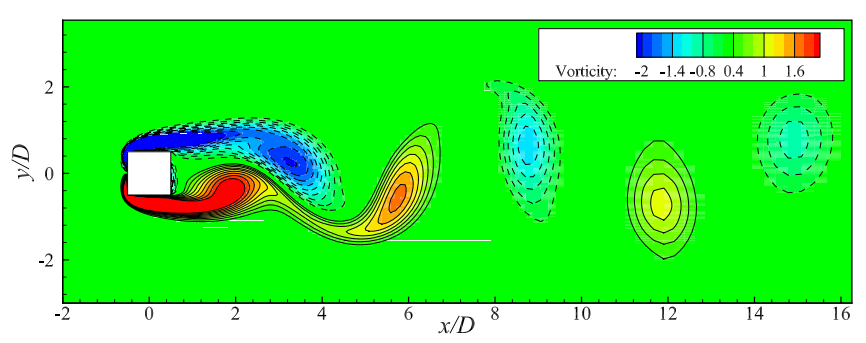

Figure 7. Instantaneous contours of the spanwise vorticity for flow past a stationary square cylinder at $\mathrm{Re}=100$.

Fig. 6 displays the time-dependent coefficients of the drag and lift force for $\mathrm{Re}=100$. The frequency of the drag coefficient oscillation is found to be twice that of the lift coefficient. This result is due to the contribution of the upper and lower alternating vortices to the drag effort. Fig. 7 shows the instantaneous contour of the spanwise vorticity, which we can see the flow patterns such as vortex shedding behind a rectangular cylinder. These numerical predictions agree well with numerical and experimental results reported by other researchers (listed in Table II).

TABLE II. COMPARISON OF FLOW VARIABLES BETWEEN THE PRESENT PREDICTIONS AND THE NUMERICAL RESULTS OBTAINED BY OTHER RESEARCHERS.

\begin{tabular}{|l|c|c|c|}
\hline \multicolumn{1}{|c|}{ Source } & $\bar{C}_{D}$ & $C_{L, r m s}$ & $S t$ \\
\hline Shen and Chan [5] & 1.464 & 0.171 & 0.146 \\
\hline Pavlov et al. [6] & 1.51 & 0.137 & 0.15 \\
\hline Sharma and Eswaran [7] & 1.494 & 0.193 & 0.149 \\
\hline Present & 1.491 & 0.192 & 0.147 \\
\hline
\end{tabular}




\section{Flow around a circular cylinder}

Both of the previous flows have involved simple Cartesian geometries and viscous boundary layers aligned with the principal coordinate directions. In this last case considered, a ghost cell immersed boundary method is used to embed a complex boundary in the Cartesian grid and simulate the timedependent flow around a circular cylinder. For Reynolds number below 47 , the flow structure remains symmetric with stationary recirculating vortices behind the cylinder. As the Reynolds umber is elevated, the symmetry breaks down and the vortex starts to shed up and down alternatively. This shedding frequency and the intensity of the vortex also increase in tandem with the elevated level of the Reynolds number.

The unbounded, uniform flow past a fixed circular cylinder with $\mathrm{Re}=40$ and $\mathrm{Re}=200$ have been calculated and the results compared with previous experimental and simulation data. The computational domain and boundary conditions are seemly to that of the flow past a square cylinder in the previous case. In this case, ghost cell immersed boundary method is used, thus permitting the use of structured Cartesian mesh to simulate flow involving complex boundaries.
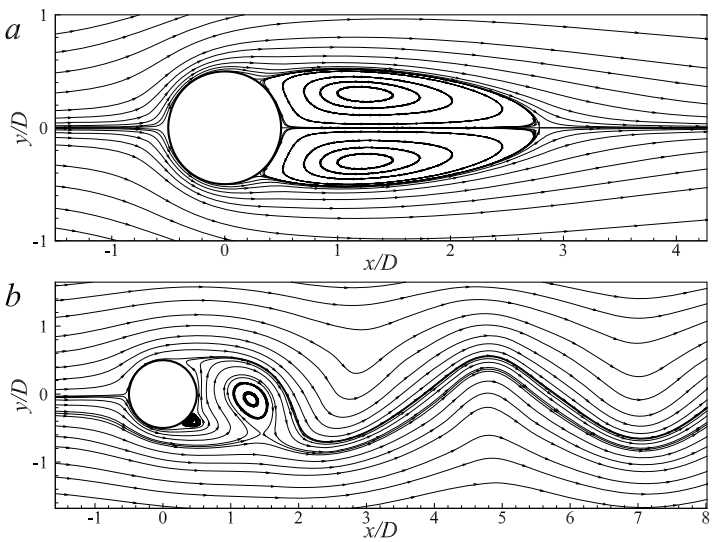

Figure 8. Streamlines for flow past a 2D stationary circular cylinder at (a) $\mathrm{Re}=40$ and (b) $\mathrm{Re}=200$.

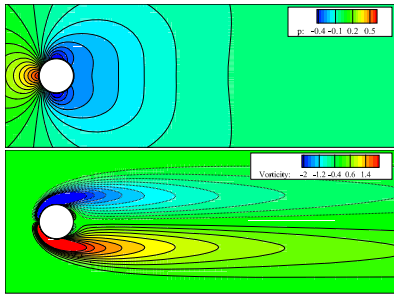

$a$

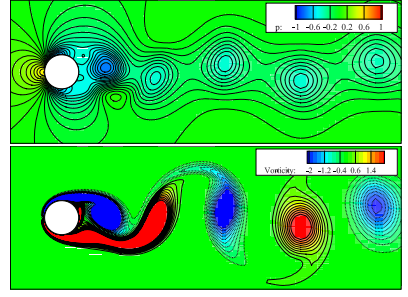

$b$
Figure 9. Pressure fields and vorticity fields for flow past a 2D stationary circular cylinder at (a) $\mathrm{Re}=40$ and (b) $\mathrm{Re}=200$.

A steady state with a pair of symmetric weak behind the circular cylinder is obtained at Reynolds number 40. For Reynolds 200 the instabilities take place. Fig. 8 shows the streamline counter and Fig. 9 shows the pressure field and vorticity field for two Reynolds numbers. It can be visualized in both figures that for Reynolds 40 the flow symmetric and for Reynolds 200 the flow becomes unstable to perturbations and leads to periodic Karman vortex shedding.
Finally, in Table III we compare the wake length, the mean drag coefficient and the vortex shedding Strouhal number predicted by the current method with some others numerical studies that have conducted 2D simulation of this flow. One can see that we obtained the close results with those numerical simulations and this further confirms the accuracy of the current method.

TABLE III. COMPARISON OF COMPUTED DRAG COEFFICIENT, WAKE LENGTH AND STROUHAL NUMBER WITH RESULTS FORM PREVIOUS 2D STATIONARY CIRCULAR CYLINDER SIMULATIONS AT RE=40 AND RE=200.

\begin{tabular}{|l|c|c|c|c|}
\hline \multirow{2}{*}{\multicolumn{1}{|c|}{ Source }} & \multicolumn{2}{c|}{$\mathbf{R e}=\mathbf{4 0}$} & \multicolumn{2}{c|}{$\mathbf{R e}=\mathbf{2 0 0}$} \\
\cline { 2 - 5 } & $\bar{C}_{D}$ & $L_{W}$ & $\bar{C}_{D}$ & $S t$ \\
\hline Berthelsen and Faltinsen [8] & 1.60 & 2.29 & 1.37 & 0.200 \\
\hline $\mathrm{Xu}$ and Wang [9] & 1.66 & 2.21 & 1.42 & 0.202 \\
\hline Calhoun [10] & 1.62 & 2.18 & - & - \\
\hline Present & 1.60 & 2.29 & 1.36 & 0.200 \\
\hline
\end{tabular}

\section{CONCLUSIONS}

The present implicit fractional step method on collocated grid has been proved to be capable of simulating incompressible viscous flows. Due to the collocated arrangement variables, the present method can be used to complex boundaries flows and extended very easily to three dimensional space.

\section{REFERENCES}

[1] J. Van-Kan, A Second-order accurate pressure-correction scheme for incompressible viscous flow, SIAM J. Sci. Stat. Comput. 7 (1986) 870891.

[2] R. Mittal, H. Dong, M. Bozkurttas, F.M. Najjar, A. Vargas, A. von Loebbecke, A versatile sharp interface immersed boundary method for incompressible flows with complex boundaries, J. Comput. Phys. 227 (2008) 4825-4852.

[3] U. Ghia, K.N. Ghia, C.T. Shin, High resolutions for incompressible flow using the Navier-Stokes equations and a multigrid method, J Comput. Phys. 48 (1982) 387-411.

[4] M-H Chung, Cartesian cut cell approach for simulating incompressible flows with rigid bodies of arbitrary shape, Comput. Fluids 35 (2006) 607-623.

[5] L. Shen, E-S Chan, Numerical simulation of fluid-structure interaction using a combined volume of fluid and immersed boundary method, Ocean Eng. 35 (2008) 939-952.

[6] A.N. Pavlov, S.S. Sazhin, R.P. Fedorenko, M.R. Heikal, A conservative finite difference method and its application for the analysis of a transient flow around a square prism, Int. J. Numer. Methods Heat Fluid Flow 10 (2000) 6-46.

[7] A. Sharma, V. Eswaran, Heat and fluid flow across a square cylinder in the two-dimensional laminar flow regime, Numer. Heat Transfer, Part A 45 (2004) 247-269.

[8] P.A. Berthelsen, O.M. Faltinsen, A local directional ghost cell approach for incompressible viscous flow problems with irregular boundaries, J. Comput. Phys. 227 (2008) 4354-4397.

[9] S. Xu, Z.J. Wang, An immersed interface method for simulation for large-eddy simulation of turbulent flows interactiong with moving boundaries, J. Comput. Phys. 215 (2006) 12-40.

[10] D. Calhoun, A Cartesian grid method for solving the two-dimensional streamfunction-vorticity equations in irregular regions, J. Comput. Phys. 176 (2002) 231-275. 